\title{
Trends in the annual incidence of carbapenem-resistant Klebsiella pneumoniae bloodstream infections: a 8-year retrospective study in a large teaching hospital in northern Italy
}

\author{
Cristiano Alicino ${ }^{1 \dagger}$, Daniele Roberto Giacobbe ${ }^{2^{*}+}$, Andrea Orsi ${ }^{1}$, Federico Tassinari ${ }^{1}$, Cecilia Trucchi ${ }^{1}$, \\ Giovanni Sarteschi ${ }^{2}$, Francesco Copello ${ }^{3}$, Valerio Del Bono ${ }^{2}$, Claudio Viscoli ${ }^{2}$ and Giancarlo Icardi ${ }^{1}$
}

\begin{abstract}
Background: Bloodstream infections (BSI) due to carbapenem-resistant (C-R) Klebsiella pneumoniae (Kp) are of global concern from both clinical and public health standpoints. This retrospective study aimed to describe C-R Kp BSI epidemiology in a large teaching hospital in northern Italy.

Methods: Between 1 January 2007 and 31 December 2014, annual incidences both of C-R Kp BSI and of carbapenem-susceptible (C-S) Kp BSI were calculated as the number of events per 10,000 patient-days. A Chi square test for linear trend was used to assess the change in the incidence of C-R Kp BSI and C-S Kp BSI over the study period. Crude 30-day mortality rates were provided both for C-R Kp BSI and for C-S Kp BSI.

Results: From 2007 to 2014, we observed 511 episodes of Kp BSI, 349 of which were caused by C-R Kp (68.3 \%). The incidence of C-R Kp BSI considerably increased from 0.04/10,000 patient-days in 2007 to 1.77/10,000 patient-days in 2014 (Chi square for trend $p<0.001$ ). The highest incidence of C-R Kp BSI was observed in intensive care units (ICUs), with a peak of 22.01 C-R Kp BSI/10,000 patient-days in 2012. A less marked but significant increase of C-S Kp BSI was also observed (Chi square for trend $p=0.004$ ). Crude 30 -day mortality was $36.1 \%$ in patients with C-R Kp BSI and $23.5 \%$ in those with C-S Kp BSI.
\end{abstract}

Conclusions: During the study period, we observed a dramatic increase in the incidence of C-R Kp BSI in our hospital. More concerted infection-control efforts are needed to contain this alarming C-R Kp diffusion.

Keywords: Incidence, Bloodstream infection, Carbapenem-resistant Klebsiella pneumoniae

\section{Background}

Bloodstream infections (BSI) due to carbapenem-resistant (C-R) Klebsiella pneumoniae (Kp) are of global concern from both clinical and public health standpoints. Indeed, these severe infections are caused by rapidly spreading clones that are frequently resistant to almost all the antibiotics used in the everyday clinical practice [1-6].

\footnotetext{
* Correspondence: daniele.roberto.giacobbe@gmail.com

${ }^{\dagger}$ Equal contributors

2Department of Health Sciences, University of Genoa and Infectious Diseases Unit, IRCCS AOU San Martino-IST teaching Hospital, L.go R. Benzi, 10-16132 Genoa, Italy Full list of author information is available at the end of the article
}

Occurring especially across Southern Europe, the rapid diffusion of C-R Kp has worryingly impressed the medical community, and several guidelines have been recently developed to reduce the spread of these difficultto-treat gram-negative rods [7-9]. In Italy, carbapenem resistance among blood $\mathrm{Kp}$ isolates has dramatically increased from $1.3 \%$ in 2009 to $29.1 \%$ in 2012 , and even higher rates of $34.3 \%$ have been reported in 2013 [10, 11]. With the aim of more clearly investigate this alarming C-R Kp diffusion, we retrospectively assessed the epidemiology of C-R Kp BSI in a large teaching hospital in northern Italy over a 8-year period. 


\section{Methods}

\section{Data collection and definitions}

We performed a retrospective study in IRCCS AOU San Martino - IST, a 1,300-beds tertiary adult acute-care teaching hospital in Genoa, Italy. Between 1 January 2007 and 31 December 2014, numbers of hospital patientdays were obtained from the digital archives of patients' clinical charts. Then, numbers of both carbapenemsusceptible (C-S) BSI and C-R Kp BSI were identified through the computerized microbiology laboratory database. The completeness and quality of laboratory data was successfully assessed by matching the hospital admission code with data obtained by clinical charts.

Only health-care associated C-R Kp BSI were considered for the analysis. According to the European Center for Disease Control and prevention (ECDC) definitions, a health-care associated C-R Kp BSI was defined as a positive blood culture collected at least $48 \mathrm{~h}$ after hospital admission, or within $48 \mathrm{~h}$ from hospital admission in those patients who had been discharged in the preceding two days [12]. For patients with multiple episodes of C-R Kp BSI, a novel event was considered as independent if occurring at least 30 days after the last positive blood culture [12].

This epidemiological analysis was performed within the Institutional surveillance of C-R Kp BSI that is periodically reported to the Regional Health Authority as a component of the Regional Plan for the Healthcareassociated infections Prevention and Control, approved by regional and national laws $[13,14]$. The study involved the analysis of existing anonymized clinical and laboratory data. An informed consent for the use of anonymized data for scientific purposes is signed by all patients admitted to IRCCS AOU San Martino - IST and included in surveillance databases. The study has been approved by the Regional Ethics Committee of Liguria Region.

\section{Microbiology}

The Vitek 2 system (bioMérieux, Marcy l'Etoile, France) was used for Kp identification and antimicrobial susceptibility testing. The interpretative breakpoints were based on the European Committee on Antimicrobial Susceptibility Testing (EUCAST) criteria (EUCAST breakpoint tables for interpretation of MICs and zone diameters, version 4.0, 2014; http://www.eucast.org). For the analysis of data, Kp isolates which were resistant to one or more carbapenems tested in our institution (i.e., ertapenem, imipenem, or meropenem) were considered to be $\mathrm{C}-\mathrm{R}$, while isolates showing full or intermediate susceptibility to all tested carbapenems were classified as C-S.

\section{Statistical analysis}

The primary study analysis aimed to establish the annual incidences of C-R Kp BSI during the study period.
Annual incidences of C-R Kp BSI with their $95 \%$ confidence intervals $(\mathrm{CI})$ were calculated as the number of events per 10,000 patient-days. In addition, annual incidences of C-R Kp BSI were also stratified by subgroups according to the ward where the diagnosis of $\mathrm{C}-\mathrm{R} \mathrm{Kp}$ BSI was made (i.e., intensive care units [ICUs], medical wards, surgical wards, or rehabilitation wards). A Chi square test for linear trend was used to assess the change in the incidence of C-R Kp BSI in our hospital over the study period.

An additional aim was to detail the overall trends in the incidence of Kp BSI. Therefore, annual incidences of health-care associated C-S Kp BSI were also calculated, by means of the methods described above. Finally, crude 30-day mortality rates were assessed both for C-R Kp BSI and for C-S Kp BSI.

All the analyses were performed using Epi-Info 7.0 (Centers for Disease Control and Prevention, CDC, Atlanta, GA, USA) and the SPSS Statistics version 20.0 (IBM Corp., Armonk, NY, USA).

\section{Results}

Between January 2007 and December 2014, we identified 511 episodes of Kp BSI, 349 of which were caused by CR Kp (68.3\%). These episodes occurred in 327 patients, of whom 19 had recurrent infections (5.8\%). The median age of patients was 68 years (interquartile range [IQR] 57-76). As many as 231/349 C-R Kp BSI occurred in males $(66.2 \%)$, thus defining a $2: 1$ male to female ratio. The median time to $\mathrm{C}-\mathrm{R} \mathrm{Kp}$ BSI diagnosis from hospital admission was 24 days (IQR 12-45), and the median length of hospital stay was 52 days (IQR 30-90).

During the study period, the overall incidence of $\mathrm{C}-\mathrm{R}$ Kp BSI was $0.92 / 10,000$ patient-days, increasing from $0.04 / 10,000$ patient-days in 2007 to $1.77 / 10,000$ patientdays in 2014 (Chi square for trend $p<0.001$ ). Detailed year-by-year incidences of C-R Kp BSI are outlined in Table 1, which also reports annual incidences of C-R Kp BSI according to wards' subgroups. As shown in the table, C-R Kp BSI were first observed in 2007, reflecting a limited cluster in ICUs. No C-R Kp BSI occurred in 2008, while they reappeared since 2009, with annual incidences increasing from $0.56 / 10,000$ patient-days in 2009 to $1.75 / 10,000$ patient-days in 2012. After a temporary decrease in 2013, the incidence of C-R Kp BSI rose again to $1.77 / 10,000$ patient-days in 2014 .

Overall, the incidence of Kp BSI (C-R plus C-S) increased from $0.50 / 10,000$ patient-days in 2007 to 2.42 / 10,000 patient-days in 2014 (Chi square for trend $p<$ 0.001). The singular trends of C-R Kp BSI and C-S Kp BSI over the study period are depicted in Fig. 1. As shown in the figure, the overall increase in the incidence of Kp BSI was mostly due to C-R Kp BSI, although a less 
Table 1 Annual incidences of health-care associated carbapenem-resistant Klebsiella pneumoniae bloodstream infections

\begin{tabular}{|c|c|c|c|c|c|c|}
\hline \multirow{3}{*}{ Year } & \multirow{3}{*}{ No. of episodes } & \multicolumn{5}{|c|}{ Incidence per 10,000 patient-days (95 \% Cl) } \\
\hline & & \multicolumn{5}{|c|}{ Ward where the diagnosis of C-R Kp BSI was made } \\
\hline & & Overall & ICU & Surgical wards & Medical wards & Rehabilitation wards \\
\hline 2007 & 2 & $0.04(0.01-0.17)$ & $1.15(0.29-4.59)$ & - & - & - \\
\hline 2008 & 0 & - & - & - & - & - \\
\hline 2009 & 27 & $0.56(0.38-0.82)$ & $12.70(8.44-19.1)$ & $0.14(0.04-0.57)$ & $0.04(0-0.28)$ & $0.60(0.09-4.27)$ \\
\hline 2010 & 39 & $0.83(0.60-1.13)$ & $17.18(11.94-24.71)$ & $0.08(0.01-0.55)$ & $0.32(0.16-0.64)$ & $0.44(0.06-3.12)$ \\
\hline 2011 & 59 & $1.22(0.94-1.57)$ & $21.06(15.40-28.81)$ & $0.69(0.36-1.32)$ & $0.36(0.19-0.69)$ & $0.60(0.02-2.39)$ \\
\hline 2012 & 83 & $1.75(1.41-2.17)$ & $22.01(16.09-30.11)$ & $1.55(0.99-2.43)$ & $0.96(0.64-1.44)$ & $0.44(0.11-1.76)$ \\
\hline 2013 & 60 & $1.30(1.01-1.68)$ & $18.17(12.98-25.42)$ & $0.61(0.29-1.29)$ & $0.72(0.44-1.17)$ & $0.48(0.15-1.48)$ \\
\hline 2014 & 79 & $1.77(1.42-2.20)$ & $18.79(13.62-25.93)$ & $0.66(0.31-1.37)$ & $1.32(0.92-1.89)$ & $0.98(0.41-2.36)$ \\
\hline
\end{tabular}

$C-R$ Kp BSI carbapenem-resistant Klebsiella pneumoniae bloodstream infections, ICU intensive care unit

marked but significant increase of C-S Kp BSI was also observed (Chi square for trend $p=0.004$ ).

Finally, crude 30-day mortality was higher in patients with C-R Kp BSI than in those with C-S Kp BSI (36.1 vs. $23.5 \%$, respectively). However, this comparison was not further considered in our analyses, since it could not be adjusted for some important clinical confounders (e.g., severity of illness, type of antibiotic therapy) that were not explored in this epidemiologic study.

\section{Discussion}

From 2007 to 2014 we noticed an impressive increase of more than $300 \%$ in the incidence of Kp BSI in our hospital, that was mostly driven by $\mathrm{C}-\mathrm{R}$ strains. This finding is of particular concern, since C-R Kp BSI carry a heavy burden in terms of morbidity and mortality [3-6].
A worrisome and persistent increase of carbapenemresistance among $\mathrm{Kp}$ isolates has been reported in Italy since 2005, with current national rates being as high as $34 \%[11,12]$. Our experience confirms this picture, since we observed a rapid increase in the incidence of C-R Kp BSI starting from 2007, that peaked at 1.75/ 10,000 patient-days in 2012. A temporary decrease was then observed in 2013, possibly because of the intensification of our hospital-based infection control measures (i.e., rapid identification of colonized and/or infected patients trough a centralized and computerized laboratorybased alert system, patient dedicated use of gowns and gloves) that occurred at that time. Starting from the same period, an active surveillance of colonized patients and their contacts was also implemented, by means of rectal swab and according to European guidelines [9].

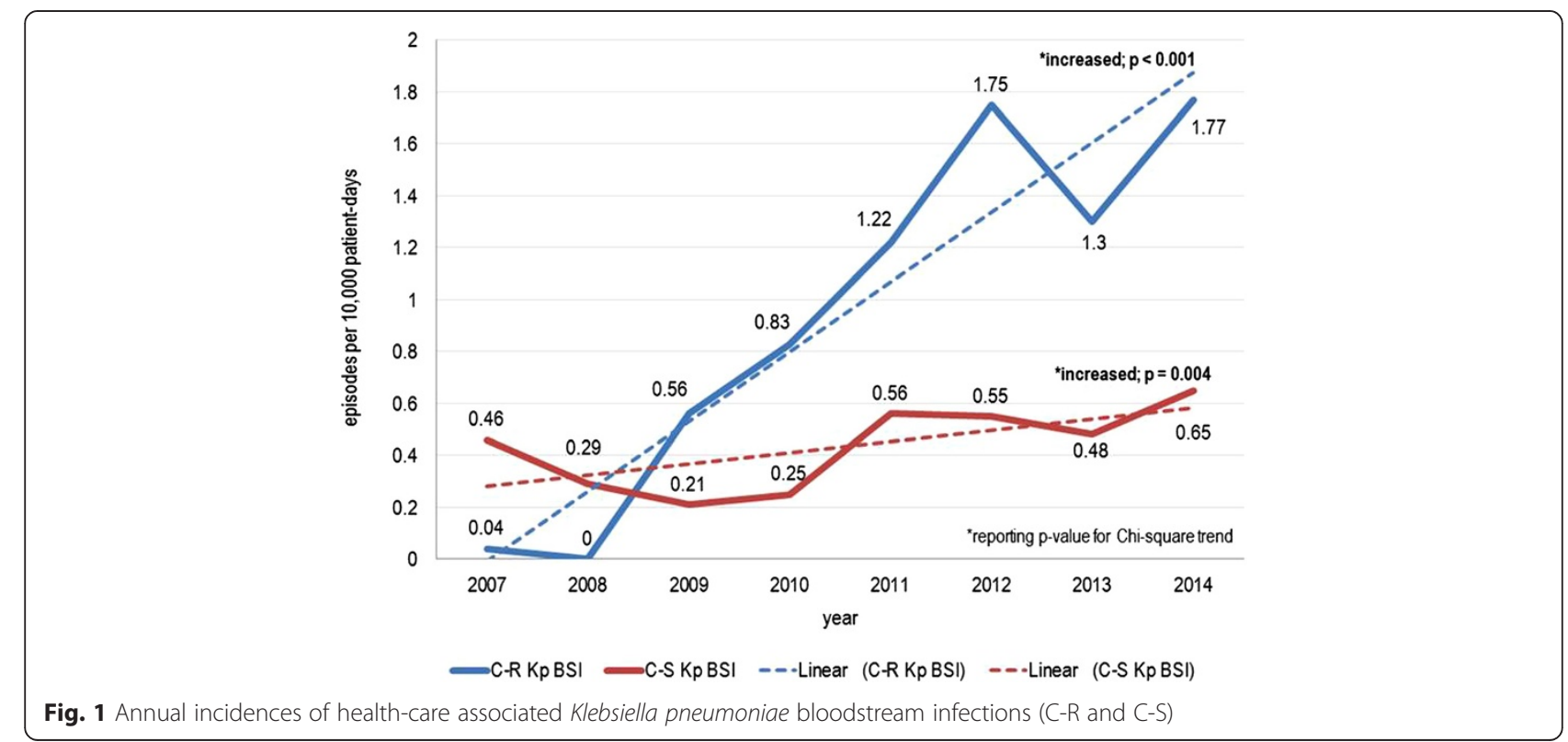


All these measures have been already proven to reduce the incidence of infections due to multidrug resistant gram negatives, including C-R Kp [15-19]. However, after the temporary decrease mentioned above, the incidence of C-R Kp BSI in our hospital rose again to $1.77 / 10,000$ patient-days in 2014. Although a lack of adherence to contention measures might have occurred in some cases, another factor possibly underlie this increase that mostly occurred outside the ICU. Indeed, we recently observed that some C-R Kp intestinal carriers in low risk wards (i.e., wards other than ICU or cancer wards) might not be considered at risk (and thus not screened) even if all recommended infection-control protocols are adopted [20]. This finding suggests that a considerable number of unknown carriers might be circulating in our hospital that are not subjected to contact precautions because of the unawareness of their status. This possibility is rather worrisome, since it could lead to an uncontrolled C-R $K p$ diffusion, thus fueling the reservoir of patients who possibly develop C-R Kp BSI [20, 21]. In such an uncontrolled endemic setting, further infection-control efforts (e.g., a dynamic redefinition of wards to adopt universal screening through regular point-prevalence surveys) might be necessary to successfully counteract the alarming spread of C-R Kp.

The present study has some important limitations. First, we could not depict the true pattern of C-R Kp diffusion, since only blood C-R Kp isolates were considered. However, C-R Kp BSI can provide a reliable picture of this diffusion, since blood cultures are collected from nearly all patients with C-R Kp BSI because of the symptomatic nature of the infection. Conversely, any possible estimation based on colonized patients would have been affected by an important selection bias due to the differences in the number of screened patients across the study years. Second, the retrospective nature of the study prevented us from investigating the degree of compliance to infectioncontrol measures during the study period, as well as the molecular characteristics of $\mathrm{C}-\mathrm{R} \mathrm{Kp}$ isolates. In this regard, a major limitation is the lack of information about both the clonal type of $\mathrm{Kp}$ isolates and their mechanisms of resistance to carbapenems. Finally, this was a single center study, and incidences of C-R Kp BSI may differ significantly across centers. Therefore, any extrapolation of our findings to other settings should be made with due caution.

\section{Conclusions}

In our hospital a dramatic increase in the incidence of C-R Kp BSI occurred from 2007 to 2014 that appeared to be only temporarily arrested by the intensification of hospital-based infection-control measures. More concerted efforts are necessary to tackle this alarming C-R Kp diffusion. Because of the dramatic shortage in antibiotics active against C-R Kp, further improvements in our infectioncontrol protocols are also of paramount importance to curtail the high number of deaths due to C-R Kp BSI.

\section{Abbreviations}

BSI: Bloodstream infections; C-R: Carbapenem-resistant; C-S: Carbapenemsusceptible; Cl: Confidence intervals; ECDC: European Center for Disease Control and prevention; EUCAST: European Committee on Antimicrobial Susceptibility Testing; Kp: Klebsiella pneumoniae; ICUs: Intensive care units; IQR: Interquartile range; MICs: Minimum inhibitory concentrations.

\section{Competing interest}

All authors report no conflicts of interest relevant to this article.

\section{Authors' contributions}

CA and DRG conceived and designed the research, collected data, cleaned and analyzed the data, and drafted and revised the paper. AO conceived and designed the research and drafted and revised the paper. FT collected and cleaned the data and revised the paper. $\subset T$ revised the paper. GS collected and cleaned the data and revised the paper. FC collected the data and revised the paper. VDB revised the paper. CV conceived and designed the research and revised the paper. Gl conceived and designed the research and revised the paper. All authors read and approved the final manuscript.

\section{Acknowledgments}

Financial support

None reported.

\section{Author details}

'Department of Health Sciences, University of Genoa and Hygiene and Infection Control Unit, IRCCS AOU San Martino-IST teaching Hospital, Genoa, Italy. ${ }^{2}$ Department of Health Sciences, University of Genoa and Infectious Diseases Unit, IRCCS AOU San Martino-IST teaching Hospital, L.go R. Benzi, 10-16132 Genoa, Italy. ${ }^{3}$ Occupational Medicine Unit, IRCCS AOU San Martino-IST teaching Hospital, Genoa, Italy.

Received: 26 February 2015 Accepted: 28 September 2015 Published online: 13 October 2015

\section{References}

1. Munoz-Price LS, Poirel L, Bonomo RA, Schwaber MJ, Daikos GL, Cormican M, et al. Clinical epidemiology of the global expansion of Klebsiella pneumoniae carbapenemases. Lancet Infect Dis. 2013;13:785-96.

2. Nordmann $P$, Dortet $L$, Poirel L. Carbapenem resistance in Enterobacteriaceae: here is the storm! Trends Mol Med. 2012;18:263-72.

3. Qureshi ZA, Paterson DL, Potoski BA, Kilayko MC, Sandovsky G, Sordillo E, et al. Treatment outcome of bacteremia due to KPC-producing Klebsiella pneumoniae: superiority of combination antimicrobial regimens. Antimicrob Agents Chemother. 2012;56:2108-13.

4. Tumbarello M, Viale P, Viscoli C, Trecarichi EM, Tumietto F, Marchese A, et al. Predictors of mortality in bloodstream infections caused by Klebsiella pneumoniae carbapenemase-producing K. pneumoniae: importance of combination therapy. Clin Infect Dis. 2012;55:943-50.

5. Zarkotou O, Pournaras S, Tselioti P, Dragoumanos V, Pitiriga V, Ranellou K, et al. Predictors of mortality in patients with bloodstream infections caused by KPC-producing Klebsiella pneumoniae and impact of appropriate antimicrobial treatment. Clin Microbiol Infect. 2011;17:1798-803.

6. Tumbarello M, Trecarichi EM, De Rosa FG, Giannella M, Giacobbe DR, Bassetti M, et al. Infections caused by KPC-producing Klebsiella pneumoniae: differences in therapy and mortality in a multicentre study. J Antimicrob Chemother. 2015;70:2133-43.

7. Centers for Disease Control and Prevention (CDC). Guidance for control of infections with carbapenem-resistant or carbapenemase-producing Enterobacteriaceae in acute care facilities. MMWR Morb Mortal Wkly Rep. 2009;58:256-60.

8. European Centre for Disease Prevention and Control. Risk assessment on the spread of carbapenemase-producing Enterobacteriaceae (CPE) through patient transfer between healthcare facilities, with special emphasis on cross-border transfer. Stockholm: ECDC; 2011. 
9. Tacconelli E, Cataldo MA, Dancer SJ, De Angelis G, Falcone M, Frank U, et al. ESCMID guidelines for the management of the infection control measures to reduce transmission of multidrug-resistant Gram-negative bacteria in hospitalized patients. Clin Microbiol Infect. 2014;20:1-55.

10. European Centre for Disease Prevention and Control. Antimicrobial resistance surveillance in Europe 2013. Annual Report of the European Antimicrobial Resistance Surveillance Network (EARS-Net). Stockholm: ECDC; 2014

11. European Centre for Disease Prevention and Control. Antimicrobial resistance surveillance in Europe 2012. Annual Report of the European Antimicrobial Resistance Surveillance Network (EARS-Net). Stockholm: ECDC; 2013.

12. HELICS Surveillance of Nosocomial Infections in Intensive Care Units protocol, version 6.1, September 2004. Available from http://ecdc.europa.eu/ en/activities/surveillance/HAl/Documents/0409_IPSE_ICU_protocol.pdf. Accessed date October 1, 2015.

13. Agenzia Regionale Sanitaria della Regione Liguria. Determina del Direttore Generale n. 125 del 19/12/2014 "Gestione di Enterobacteriaceae resistenti ai carbapeneminelle Strutture Sanitarie". Available from http:/www.arsliguria. it/index.php?option=com_docman\&task=doc_download\&gid=1284\& Itemid=240.

14. Ministero della Salute. Circolare del Ministero della Salute n. 4968 del 26/02/ 2013 "Sorveglianza, e controllo delle infezioni da batteri produttori di carbapenemasi (CPE). Available from http://www.trovanorme.salute.gov.it/ norme/renderNormsanPdfijsessionid=f7-y3XA5uvri021r5t2+Kw_.sgc3-prdsal?anno $=0 \&$ codLeg $=45499 \&$ parte $=1 \% 20 \&$ serie $=$.

15. Gbaguidi-Haore H, Legast S, Thouverez M, Bertrand X, Talon D. Ecological study of the effectiveness of isolation precautions in the management of hospitalized patients colonized or infected with Acinetobacter baumannii. Infect Control Hosp Epidemiol. 2008;29:1118-23.

16. Kochar S, Sheard T, Sharma R, Hui A, Tolentino E, Allen G, et al. Success of an infection control program to reduce the spread of carbapenem-resistant Klebsiella pneumoniae. Infect Control Hosp Epidemiol. 2009;30:447-52.

17. Vonberg RP, Wolter A, Chaberny IF, Kola A, Ziesing S, Suerbaum S, et al. Epidemiology of multi-drug-resistant gram-negative bacteria: data from an university hospital over a 36-month period. Int J Hyg Environ Health. 2008:211:251-57.

18. Viale P, Tumietto F, Giannella M, Bartoletti M, Tedeschi S, Ambretti S, et al. Impact of a hospital-wide multifaceted programme for reducing carbapenem-resistant Enterobacteriaceae infections in a large teaching hospital in northern Italy. Clin Microbiol Infect. 2015;21:242-7.

19. Hayden MK, Lin MY, Lolans K, Weiner S, Blom D, Moore NM, et al. Prevention of Colonization and Infection by Klebsiella pneumoniae Carbapenemase-Producing Enterobacteriaceae in Long-term Acute-Care Hospitals. Clin Infect Dis. 2015;60:1153-61.

20. Giacobbe DR, Del Bono V, Marchese A, Viscoli C. Early carbapenem-resistant Klebsiella pneumoniae bacteraemia: should we expand the screening? Clin Microbiol Infect. 2014;20:01157-8.

21. Giannella M, Trecarichi EM, De Rosa FG, Del Bono V, Bassetti M, Lewis RE, et al. Risk factors for carbapenem-resistant Klebsiella pneumoniae bloodstream infection among rectal carriers: a prospective observational multicenter study. Clin Microbiol Infect. 2014;20:1357-62.

\section{Submit your next manuscript to BioMed Central and take full advantage of:}

- Convenient online submission

- Thorough peer review

- No space constraints or color figure charges

- Immediate publication on acceptance

- Inclusion in PubMed, CAS, Scopus and Google Scholar

- Research which is freely available for redistribution 